\title{
Melatonin Plays a Protective Role by Regulating miR-26a-5p-NRSF and JAK2-STAT3 Pathway to Improve Autophagy, Inflammation and Oxidative Stress of Cerebral Ischemia-Reperfusion Injury
}

This article was published in the following Dove Press journal:

Drug Design, Development and Therapy

\author{
Bo Yang' \\ Li-E Zang' \\ Jing-Wen Cui ${ }^{2}$ \\ Ming-Yuan Zhang ${ }^{2}$ \\ Xue $\mathrm{Ma}^{3}$ \\ Lin-Lin $\mathrm{Wei}^{4}$
}

'Department of Neurology, The First Affiliated Hospital of Jinzhou Medical University, Jinzhou, Liaoning, People's Republic of China; ${ }^{2}$ Department of Neurosurgery, The First Affiliated Hospital of Jinzhou Medical University, Jinzhou, Liaoning, People's Republic of China; ${ }^{3}$ Department of Emergency, The First Affiliated Hospital of Jinzhou

Medical University, Jinzhou, Liaoning, People's Republic of China; ${ }^{4}$ Department of Gynaecology, The First Affiliated Hospital of Jinzhou Medical University, Jinzhou, Liaoning, People's Republic of China
Correspondence: Lin-Lin Wei Department of Gynaecology, The First Affiliated Hospital of Jinzhou Medical University, No. 2, Section 5, Renmin Street, Jinzhou, Liaoning 121000 , People's Republic of China

Tel +86-4l6-4605293

Email linlinweii@hotmail.com
Background: Melatonin (MT) has potential protective effect on cerebral ischemiareperfusion injury (CIRI), but its underlying regulatory mechanism has not been identified. Purpose: This study aimed to explore the role of miR-26a-5p-neuron-restrictive silencing factor (NRSF/REST), Janus kinase-2 (JAK2)-signal transducer and activator of transcription3 (STAT3) pathway in the protection mechanism of MT against CIRI in vivo and in vitro.

Methods: Sprague Dawley rats were induced with ischemia-reperfusion (IR) in vivo model; PC12 cells were induced with oxygen-glucose deprivation/reperfusion (OGD/R) in vitro model; and MT intervention was conducted before the model was established. The effect of MT on autophagy factors (LC3II/LC3I, P62), inflammatory factors (TNF- $\alpha$, IL-6, IL-10) and oxidative stress indexes (MDA, GSHPx, SOD) was explored, and then the above three indexes were determined by real-time quantitative PCR, ELISA, and detection kit corresponding to oxidative stress indexes. The neuroprotective effect of MT pretreatment on brain IR injury was evaluated by neurological deficit scores and TUNEL method. The levels of miR-26a-5p and NRSF were detected by real-time quantitative PCR and Western blot, and the interaction between them was evaluated by dual luciferase report. The role of JAK2-STAT3 pathway in MT protection mechanism was verified by pathway blocker (AG490) and Western blot.

Results: MT pretreatment can significantly reduce neurological deficit score and neuronal apoptosis, inhibit CIRI autophagy, inflammation and oxidative stress in vivo and in vitro, reduce LC3II/LC3I, TNF- $\alpha$, IL-6, MDA and increase P62, IL-10, GSHPx, SOD. Further analysis identifies that downregulating miR-26a-5p or upregulating NRSF can eliminate the protective effect of MT, and NRSF is the direct target of miR-26a-5p. The protective effect of MT can also be eliminated under AG490 intervention.

Conclusion: MT plays a protective role by regulating miR-26a-5p-NRSF and JAK2-STAT3 pathway to improve CIRI autophagy, inflammation and oxidative stress.

Keywords: cerebral ischemia-reperfusion injury, melatonin, miR-26a-5p, NRSF, JAK2-STAT3

\section{Introduction}

Cerebral ischemia-reperfusion injury (CIRI) is a central nervous system disease and a vital cause of ischemic stroke. ${ }^{1,2}$ The data show that ischemic stroke is the typical representative of stroke (87\%), while global stroke cases amount to about 15 million cases and death cases amount to about 5.8 million cases. ${ }^{3}$ Therefore, understanding the pathological mechanism of CIRI is quite valuable for the treatment and prevention of ischemic stroke. 
The pathological mechanism of CIRI is complex, involving cell death (including apoptosis and autophagy), inflammation, oxidative stress, etc. ${ }^{4,5}$ Melatonin (MT), as a neurohormone secreted by the pineal gland, has the antiCIRI characteristics of anti-apoptosis, anti-inflammation and anti-oxidative stress, and plays an important neuroprotective role in CIRI. For example, CIRI can be alleviated by upregulating SIRT3 expression. ${ }^{6-8}$ There are also reports that ranitidine, developed based on MT, is a selective MTreceptor agonist, which can alleviate acute and chronic CIRI by inhibiting autophagy in the cortex around an infarction. ${ }^{9}$ Although there is more and more research on the protective effect of MT, ${ }^{10,11}$ there is little research on miRNA-mRNA axis and signal pathway regulation in MT anti-CIRI protective mechanism at present. It is understood that miRNA is a micro noncoding physiological and pathological regulator, which can mediate CIRI pathological process through targeted regulation of mRNA stability or translation efficiency. ${ }^{12}$ As a member of miRNA, miR-26a$5 p$ cannot only respond to the efficacy of multiple sclerosis that is a central nervous system disease, but also reduce CIRI by inhibiting neuronal apoptosis after upregulation. ${ }^{13,14} \mathrm{We}$ found a potential binding site between miR-26a-5p and neuron-restrictive silencing factor (NRSF/REST) on the online target gene prediction website. NRSF is associated with remodeling of nerve genes and neurodegeneration. Downregulating its expression can improve CIRI process in rats by inhibiting apoptosis, reducing cortical infarct volume and enhancing expression of synaptic plasticity genes. ${ }^{15}$ Moreover, we also found that it mediated Janus kinase-2 (JAK2)-signal transducer and activator of transcription-3 (STAT3) pathway in lung cancer cells to regulate tumor metastasis. ${ }^{16}$ It is understood that JAK2-STAT3 pathway can also activate epidermal growth factor-related anti-CIRI protection mechanism, and the activation of this pathway may be a therapeutic target of anti-CIRI. ${ }^{17}$

In this study, we explored the improvement of MT on autophagy, inflammation and oxidative stress in CIRI, and analyzed the role of miR-26a-5p-NRSF and JAK2-STAT3 pathway in MT anti-CIRI protection mechanism, hoping to provide new clues for treating ischemic stroke.

\section{Materials and Methods}

\section{Animal Model in vivo}

We bought 40 male Sprague Dawley rats (Focus Biotechnology Co., Ltd, Guangzhou, China) weighing $(270+20) \mathrm{g}$, and fed them $12 \mathrm{~h}$ in a dark/light cycle environment with temperature of $25^{\circ} \mathrm{C}$, humidity of $60 \%$, providing food and water. Rats were divided into Sham group, CIRI group, CIRI+vehicle group and CIRI + MT group, with 10 rats in each group. The CIRI model was constructed as described above: First, the rat brain was occluded by longa suture (MCAO) to simulate transient cerebral ischemia, and the anesthesia state of the rat was maintained by chloral hydrate $(0.3 \mathrm{~mL} / 100 \mathrm{~g}){ }^{18}$ Two hours after blood flow occlusion in rats, thrombus was removed for reperfusion. The sham operation group was similar to CIRI except that no screws were inserted. In CIRI+MT group, MT $(5 \mathrm{mg} / \mathrm{kg})$ was given intravenously to rats before ischemia, ${ }^{19}$ while other groups were given equal volumes of PEG saline. The neurological deficit scores were evaluated $24 \mathrm{~h}$ after reperfusion in rats. Euthanasia (direct cervical dislocation) was performed $24 \mathrm{~h}$ after operation and brain tissue was collected and stored at $-70^{\circ} \mathrm{C}$ for later use. Our animal experiments were all conducted in the experimental animal center of Jinzhou Medical University. This study has been approved by the experimental animal ethics committee of this university (ethics review no. 2,020,018), and the experiments were strictly in line with the guidelines.

\section{Neurological Deficit Scores}

As mentioned earlier, the neurological deficit scoring system was adapted to score mainly from five senses and motor function, ${ }^{20}$ with a score of $0-100$. The degree of neurological deficit was proportional to the score.

\section{Neuronal Apoptosis is Measured via TUNEL Method}

The experiment employed TUNEL test kit (Qiming Biotech Co., Ltd, Shanghai, China, OX02752). Rat brain tissue was fixed $20 \mathrm{~min}$ on glass slides with $4 \%$ paraformaldehyde ice cold and permeabilized for five minutes with $0.1 \%(\mathrm{v} / \mathrm{v})$ Triton $^{\mathrm{TM}} \mathrm{X}-100$. After that, the slide and TUNEL incubation mixture were incubated in a dark and humid place $\left(37^{\circ} \mathrm{C}, 1.5 \mathrm{~h}\right)$ and stained with DAPI. Finally, TUNEL apoptotic (positive) cells were observed and counted by fluorescence microscope.

\section{Apoptosis Levels are Determined by Flow Cytometry}

Apoptosis levels were detected through apoptosis detection kit (Sanshu Biotechnology Co., Ltd, Shanghai, China, BYT0037) and flow cytometry. Cells were treated as 
follows: the cells were digested by trypsin (Jinsui Biotechnology Co., Ltd, Shanghai, China, J36530), prepared to cell suspension, inoculated on a six-well plate, and cultured for $24 \mathrm{~h}$ in serum-free DMEM (Beinuo Biotechnology Co., Ltd, Shanghai, China, Amresco N676-6X5ML). Next, the culture medium was removed and the cells were washed three times with phosphate buffer (Hengfei Biotechnology Co., Ltd, Shanghai, China, R22127). Fluorescent generator was added and they were incubated $15 \mathrm{~min}$ in the dark. Finally, the apoptosis rate was measured by FACSCanto II flow cytometry ( Biomerry Biotechnology Co., Ltd., Beijing, China).

\section{In vitro Model of $\mathrm{PCI} 2$ Cells}

PC12 cell lines (Guandao Bioengineering Co., Ltd, Shanghai, China, DA-C6046) were purchased and cultured in high-sugar DMEM medium containing 10\% FBS and 7.5\% horse serum (Lvyuan Bio-technology Co., Ltd, Beijing, China, PM150210B). The environmental conditions in the incubator were set at $37^{\circ} \mathrm{C}, 5 \% \mathrm{CO}_{2}$, and 10 $\mathrm{nM}$ nerve growth factor (Wines Da Industrial Co., Ltd, Shanghai, China, SXR051) was added to induce PC12 cells to differentiate into neuron cells. PC12 cells were divided into control group, OGD/R group, OGD/R+vehicle group and OGD/R+MT group. The oxygen glucose deprivation/reperfusion (OGD/R) model was constructed according to the above research: PC12 was treated $30 \mathrm{~min}$ with MT (50 $\mu \mathrm{M})$ or $0.1 \%$ DMSO (BJ-RD553, Shanghai, China), the collected PC12 was placed in OGD medium, Hank's balanced salt solution (HBSS) (30 min) was bubbled with nitrogen (Sangon Bioengineering Co., Ltd, Shanghai, China, A002064-0001) to consume internal and external glucose and oxygen, and then the PC12 was cultured for $24 \mathrm{~h}$ in a normal culture environment to reoxygenation. ${ }^{19}$

\section{Cell Transfection}

Based on the experimental purpose, the transfectants include miR-26a-5p inhibitor and corresponding negative control (inhibitor-NC), targeted over-expression NRSF (NRSF) and corresponding negative control (NC). Transfection agents were transfected into cells by transfection kits (Even Bridge Biotechnology Co., Ltd, Shanghai, China, K482001), and then cells transfected for $48 \mathrm{~h}$ were collected for subsequent experiments. The operation steps were strictly carried out in accordance with the kit instructions.
In addition, subsequent experiments will also apply JAK2 inhibitor AG490 (5 $\mu \mathrm{M})$ (Hengfei Biotechnology Co., Ltd, Shanghai, China, a1321) to PC12 cells to verify whether JAK2-STAT3 pathway participates in MT's protection mechanism in CIRI. ${ }^{21}$

\section{RT-PCR}

As described here previously, ${ }^{22}$ total RNA was extracted from rat cortex and PC12 cells by total RNA isolation kit (Even Bridge Biotechnology Co., Ltd A27828), amplified by PCR instrument (Yihui Biotechnology Co., Ltd, Shanghai, China, 1,852,196), and detected by mRNA and miRNA qPCR detection kit (Zhen Shanghai and Shanghai Industrial Co., Ltd, Shanghai, China, HZ10017). Primer design was completed by Shanghai Yingbai Biotechnology Co., Ltd. Among them, mRNA employed $\beta$-Actin as internal reference and miRNA employed U6 as internal reference. All data were analyzed via $2^{-\Delta \Delta c t}$.

\section{Western Blot Analysis}

Part of the brain tissue or PC12 cells were placed in cold lysis buffer (Cloud-Clone Diagnostic Reagents Institute Co., Ltd, Wuhan, China, IS007), centrifuged $10 \mathrm{~min}$ at $1500 \square \mathrm{g}$, $4^{\circ} \mathrm{C}$, and the protein concentration was determined using Bradford Protein Quantitative Kit (Hengfei Biotechnology Co., Ltd, Shanghai, China, PT0002). The precipitate was denatured by SDS sample loading buffer and separated by $10 \%$ SDS-PAGE. The protein was transferred to the membrane and incubated at $4^{\circ} \mathrm{C}$ all night, incubated four one hour with the blocking solution at room temperature, and then washed and incubated for two hours with primary antibody at room temperature. The primary antibody included NRSF and $\beta$-Actin. The membrane was washed again and incubated for two hours with a secondary antibody. Antibodies were all purchased from Shanghai Yanqi Biotechnology Co., Ltd. Finally, protein bands were analyzed by chemiluminescence kits (Dingguo Changsheng Biotechnology Co., Ltd, Beijing, China, ECL-0013).

\section{Enzyme-Linked Immunosorbent Assay (ELISA)}

As mentioned above, ${ }^{23}$ the pro-inflammatory factors (TNF- $\alpha$, IL-6) and anti-inflammatory factors (IL-10) in serum or PC12 cells in rat hippocampus were determined by ELISA kits (Hengfei Biotechnology Co., Ltd, CSBE08055r-1, CSB-E04640r-1, CSB-E11987r-1), strictly in line with the operating instructions, and finally the 
absorbance was measured at $450 \mathrm{~nm}$ by microplate reader (Image Trading Co., Ltd, Beijing, China, 21,261,000).

\section{Determination of Oxidative Stress Indexes}

The peroxide indexes (MDA) and antioxidant indexes (GSHPx, SOD) of rat brain tissue and PC12 cells were detected by respective corresponding detection kits (Jingkang Bioengineering Co., Ltd, Shanghai, China, JK(a)-2197, JK-(a)-2396, JK-(a)-2293) in strict accordance with the instructions.

\section{Statistical Analysis}

Our data were expressed by mean \pm SD. All experiments were repeated three times and data were analyzed by Prism. Data differences between two groups were analyzed via independent-samples $t$-test, and those among groups were compared by one-way analysis of variance (ANOVA) and LSD- $t$-test. $P<0.05$ was regarded as the difference with statistical significance.

\section{Results}

\section{MT Can Reduce Neurological Deficit Score and Neuronal Apoptosis}

In order to understand the neuroprotective effect of MT on CIRI rats, we have carried out relevant verification. The results showed that compared with Sham group rats, CIRI group rats had significantly higher neurological deficit score and neuronal apoptosis, while MT intervention significantly reduced the above indicators. The above results revealed that MT could restore the neural function of CIRI rats by reducing the number of neuronal apoptosis (Figure 1).

\section{MT Can Inhibit Autophagy, Inflammation and Oxidative Stress in vivo Models}

Autophagy, inflammation and oxidative stress were the main pathological mechanisms of CIRI. We explored the anti-CIRI mechanism of MT in vivo from the above three angles. The data showed that CIRI rats had autophagy, inflammation and oxidative stress. However, after MT intervention, the levels of LC3II/LC3I, TNF- $\alpha$, IL-6 and MDA reduced obviously, while the levels of P62, IL-10, GSHPx and SOD increased markedly. The above analysis identifies that MT can improve the pathological changes in CIRI rats by inhibiting autophagy, inflammation and oxidative stress, thus playing a neuroprotective role (Figure 2).

\section{MT Can Inhibit Autophagy, Inflammation and Oxidative Stress in vitro Model}

In order to explore the mechanism of MT protection in vitro, we also established OGD/R in vitro model, and analyzed from the above three aspects. The results showed that the apoptosis rate of neurons in $\mathrm{OGD} / \mathrm{R}$ in vitro model was significantly higher, while MT could reduce the level of apoptosis markedly. In addition, the $\mathrm{OGD} / \mathrm{R}$ in vitro model also showed autophagy, inflammation and oxidative stress similar to those mentioned above. After MT intervention, the levels of LC3II/LC3I, TNF- $\alpha$, IL-6 and MDA were dramatically inhibited, while the levels of P62, IL-10, GSHPx and SOD were obviously enhanced. These results signified that MT could improve autophagy, inflammation and oxidative stress in vivo

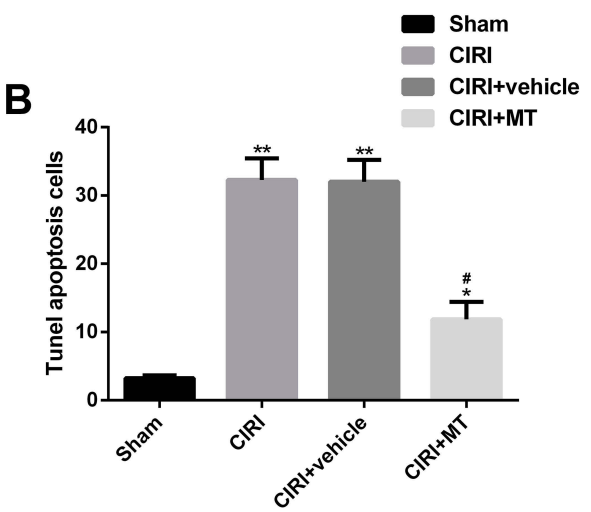

Figure I Effect of MT on neurological deficit score and neuronal apoptosis in CIRI rats. (A) MT significantly reduces the neurological deficit score of CIRI rats. (B) MT significantly reduces neuronal apoptosis cells in CIRI rats.

Notes: Compared with sham, *represents $P<0.05$ and **represents $P<0.01$; compared with CIRI, ${ }^{*}$ represents $P<0.05$.

Abbreviations: MT, melatonin; CIRI, cerebral ischemia-reperfusion injury. 

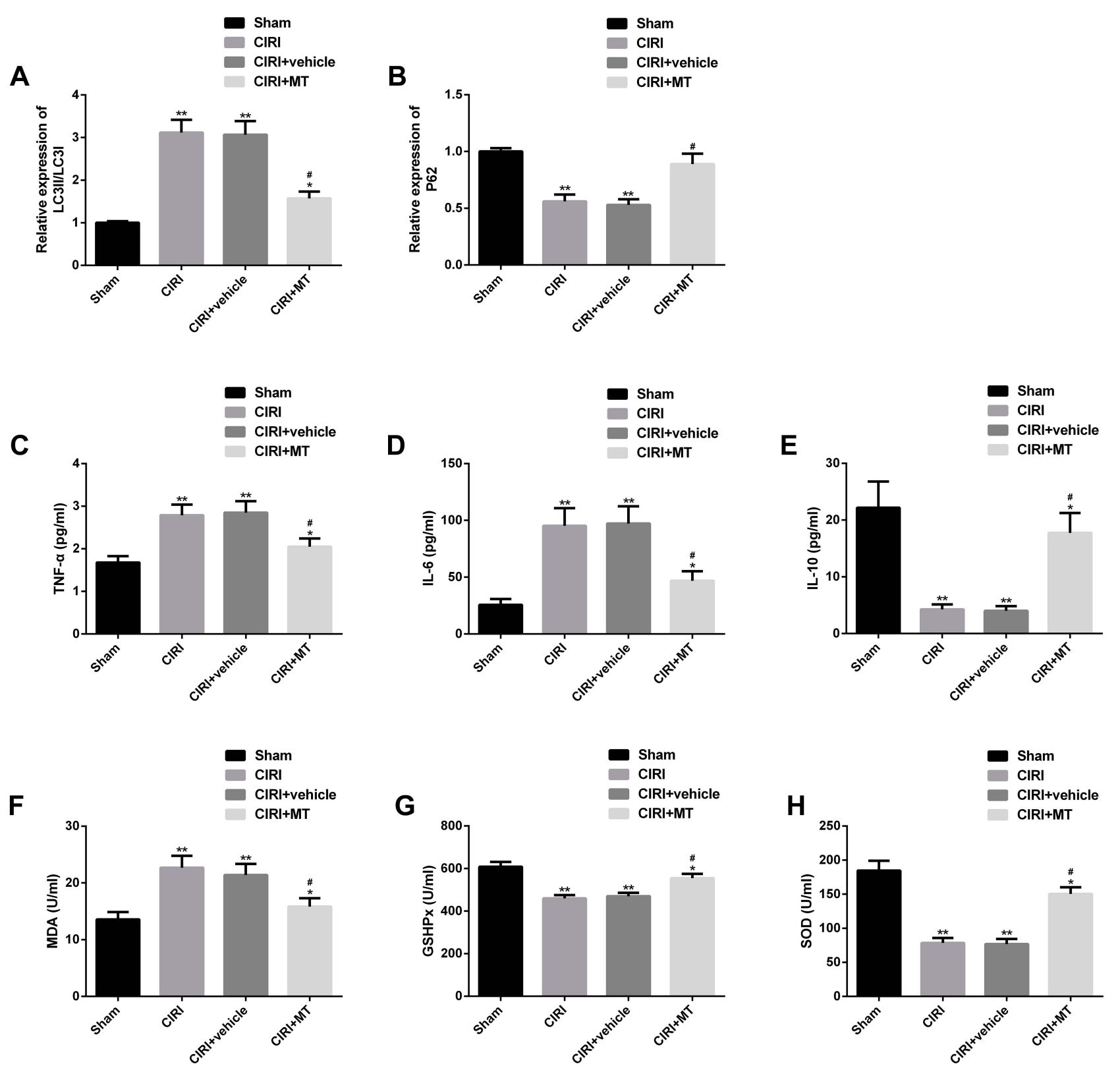

Figure 2 Effect of MT on autophagy, inflammation and oxidative stress in CIRI rats. (A-B) Effect of MT on the levels of autophagy factors LC3II/LC3I and P62 in CIRI rats; (C-E) Effect of MT on the concentrations of TNF- $\alpha$, IL-6 and IL-I0 in CIRI rats; (F-H) Effects of MT on oxidative stress indexes MDA, GSHPx and SOD in CIRI rats. Notes: Compared with sham, *represents $P<0.05$ and **represents $P<0.01$; compared with CIRI, " represents $P<0.05$.

Abbreviations: MT, melatonin; CIRI, cerebral ischemia-reperfusion injury; LC3, light chain 3; P62, sequestosome I; TNF, tumor necrosis factor; IL, interleukin; MDA, malondialdehyde; GSHPx, glutathione peroxidase; SOD, superoxide dismutase.

and in vitro, thus improving neuronal cell apoptosis and protecting the nervous function of the body (Figure 3).

\section{MT Can Regulate miR-26a-5p and NRSF in $\mathrm{CIRI}$ in vivo and in vitro Models}

In order to explore the molecular mechanism of MT's protective effect on CIRI in vivo and in vitro, we explored the effect of MT on miR-26a-5p and NRSF. We found that
CIRI in vivo and in vitro models showed significantly downregulated miR-26a-5p and upregulated NRSF (expression and protein level) compared with corresponding controls, while miR-26a-5p and NRSF showed marked improvement in different degrees after MT intervention. The above results suggest that the anti-CIRI protection mechanism of MT may be related to the regulation of miR-26a-5p and NRSF (Figure 4). 

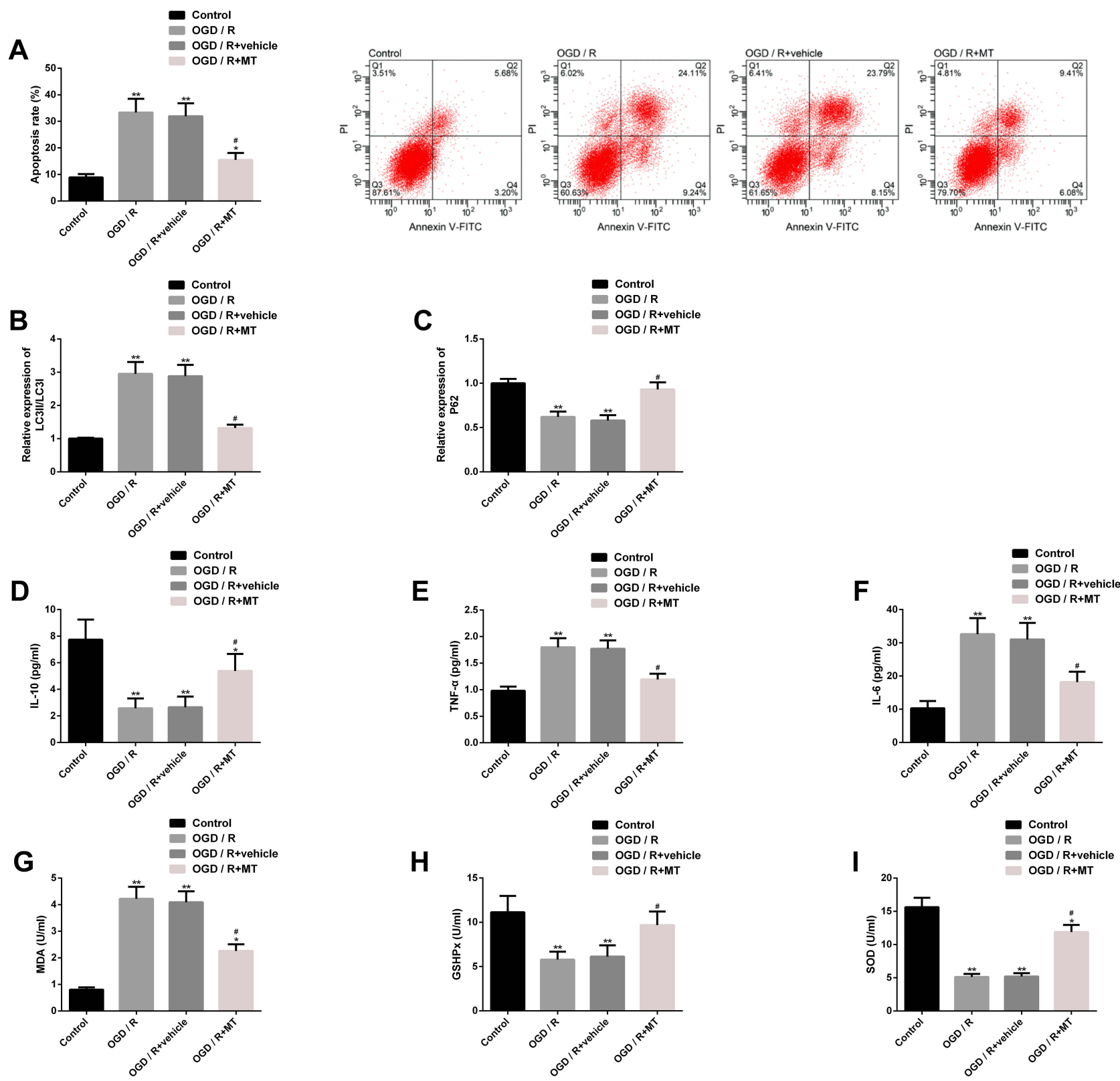

Figure 3 Effect of MT on OGD/R in vitro model. (A) Effect of MT on apoptosis of neurons in OGD/R in vitro model and its flow cytometry; (B-C) Effect of MT on the levels of autophagy factors LC3II/LC3I and P62 protein in OGD/R in vitro model and its protein map; (D-F) Effect of MT on TNF- $\alpha$, IL-6, IL-I0 concentration in OGD/R model in vitro; (G-I) Effect of MT on oxidative stress indexes MDA, GSHPx and SOD levels of OGD/R in vitro model.

Notes: Compared with control, *represents $P<0.05$ and **represents $P<0.01$; compared with $O G D / R$, ${ }^{*}$ represents $P<0.05$.

Abbreviations: MT, melatonin; OGD/R, oxygen-glucose deprivation/reperfusion; PI, propidium iodide; FITC, fluorescein isothiocyanate; LC3, light chain 3; P62, sequestosome I; TNF, tumor necrosis factor; IL, interleukin; MDA, malondialdehyde; GSHPx, glutathione peroxidase; SOD, superoxide dismutase.

\section{Downregulating miR-26a-5p Can Eliminate the Protective Effect of MT in vitro}

We further explored the role of miR-26a-5p in the in vitro protection mechanism of MT, and achieved remarkable downregulation of miR-26a-5p by transfection of miR-26a-5p inhibitors. The results revealed that downregulating miR-26a-5p could significantly eliminate the improvement of MT on neuronal cell apoptosis, autophagy, inflammation and oxidative stress. This suggests that downregulating miR-26a-5p will significantly interfere with the in vitro protection of MT and even eliminate the in vitro protection of MT (Figure 5). 


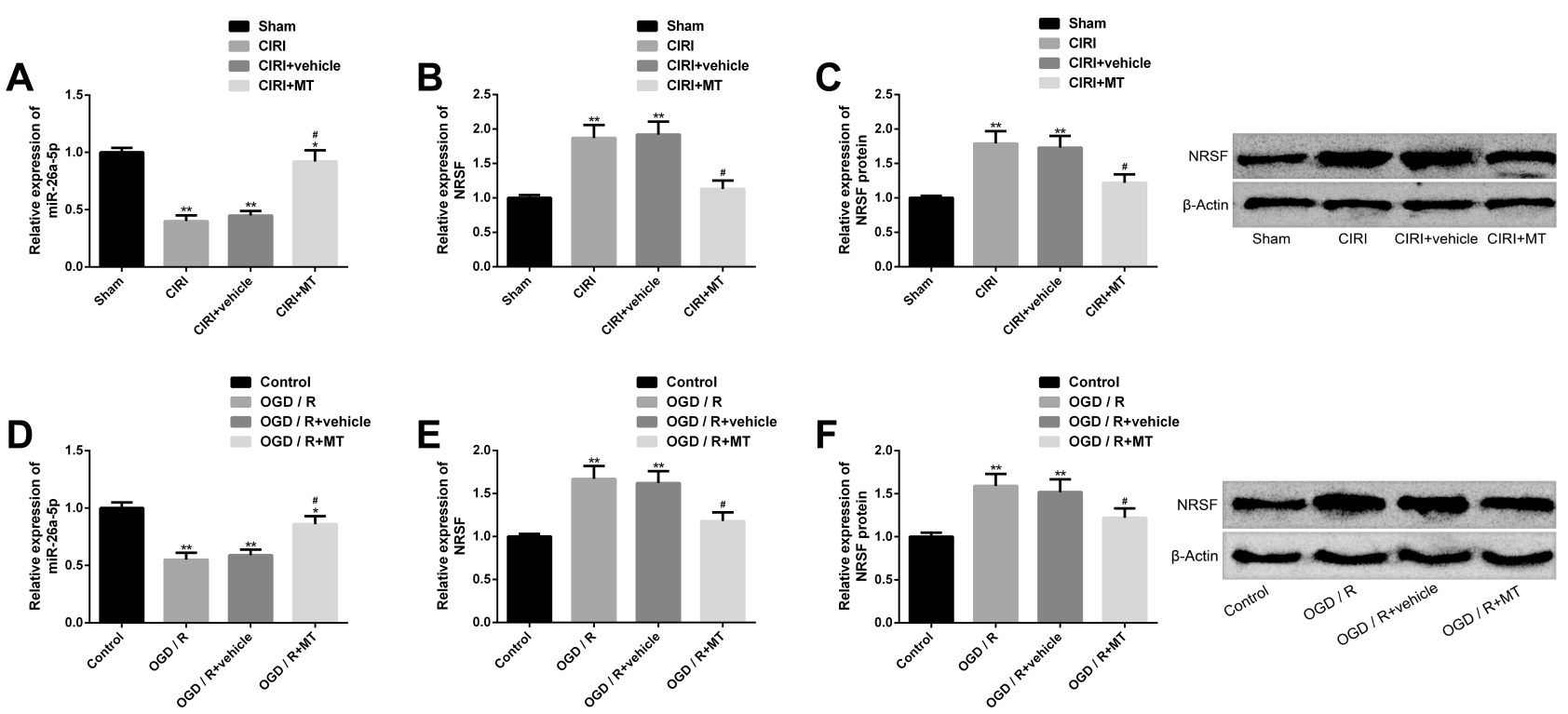

Figure 4 Effect of MT on miR-26a-5p and NRSF in CIRI in vitro and in vivo models. (A) miR-26a-5p is significantly downregulated in CIRI in vivo model, while MT can restore miR-26a-5p expression. (B-C) The expression of NRSF and protein level in CIRI in vivo model are significantly upregulated, while MT can silence NRSF and its protein map. (D) miR-26a-5p is significantly downregulated in the OGD/R in vitro model, and MT can upregulate the miR-26a-5p expression. (E-F) In vitro model of OGD/ R, NRSF expression and protein level are significantly upregulated, MT can silence NRSF and its protein map.

Notes: Compared with sham or control, *represents $P<0.05$ and **epresents $P<0.01$; compared with $C I R I$ or $O G D / R$, ${ }^{\#}$ represents $P<0.05$.

Abbreviations: MT, melatonin; CIRI, cerebral ischemia-reperfusion injury; NRSF, neuron-restrictive silencing factor; miR, microRNA; OGD/R, oxygen-glucose deprivation/ reperfusion; LC3, light chain 3; P62, sequestosome I; TNF, tumor necrosis factor; IL, interleukin; MDA, malondialdehyde; GSHPx, glutathione peroxidase; SOD, superoxide dismutase.

\section{Upregulating NRSF Can Eliminate the Protective Effect of MT in vitro}

We also explored NRSF's role in the in vitro protection mechanism of MT, and achieved marked upregulation of NRSF by transfection of NRSF. We found that the effect of upregulating NRSF was similar to downregulating miR-26a$5 p$, which threatened the in vitro protection of MT and almost eliminated the suppression of MT on neuronal apoptosis, autophagy, inflammation and oxidative stress. The above results show that the in vitro protective mechanism of MT is affected by NRSF, which mediates the protective effect (Figure 6).

\section{NRSF is a Downstream Target of miR-26a-5p}

We found the potential target sites of NRSF and miR26a-5p through TargetsCan (http://www.targetscan.org/ vert 72/). The dual luciferase report found that miR26a-5p inhibitor only significantly increased NRSF-Wt, but had no remarkable effect on NRSF-Mut. Further analysis found that miR-26a-5p inhibitor could significantly upregulate NRSF expression and protein level. The above analysis shows that NRSF, as a downstream target of miR-26a-5p, is negatively regulated by NRSF (Figure 7).

\section{MT May Exert Anti-CIRI Protection Mechanism by Activating JAK2-STAT3 Pathway}

The activation of JAK2-STAT3 pathway played a crucial role in the anti-CIRI protection mechanism. Our research showed that MT intervention could activate this pathway by significantly increasing the protein levels of p-JAK2 and p-STAT3, while the above protein levels reduced dramatically under the action of pathway inhibitor AG490. In addition, AG490 could also dramatically eliminate the anti-apoptosis effect of MT. The above results indicate that the anti-CIRI protection mechanism of MT is tied to the activation of JAK2-STAT3 pathway (Figure 8).

\section{Discussion}

CIRI is a key factor that causes ischemic stroke. It is quite significant to study the pathological mechanism and potential therapeutic drugs of CIRI for reducing ischemic stroke-related deaths. ${ }^{24}$ MT is a recognized antiinflammatory and antioxidant multi-effect molecule. Its pharmacological effects can be exerted in various nervous 

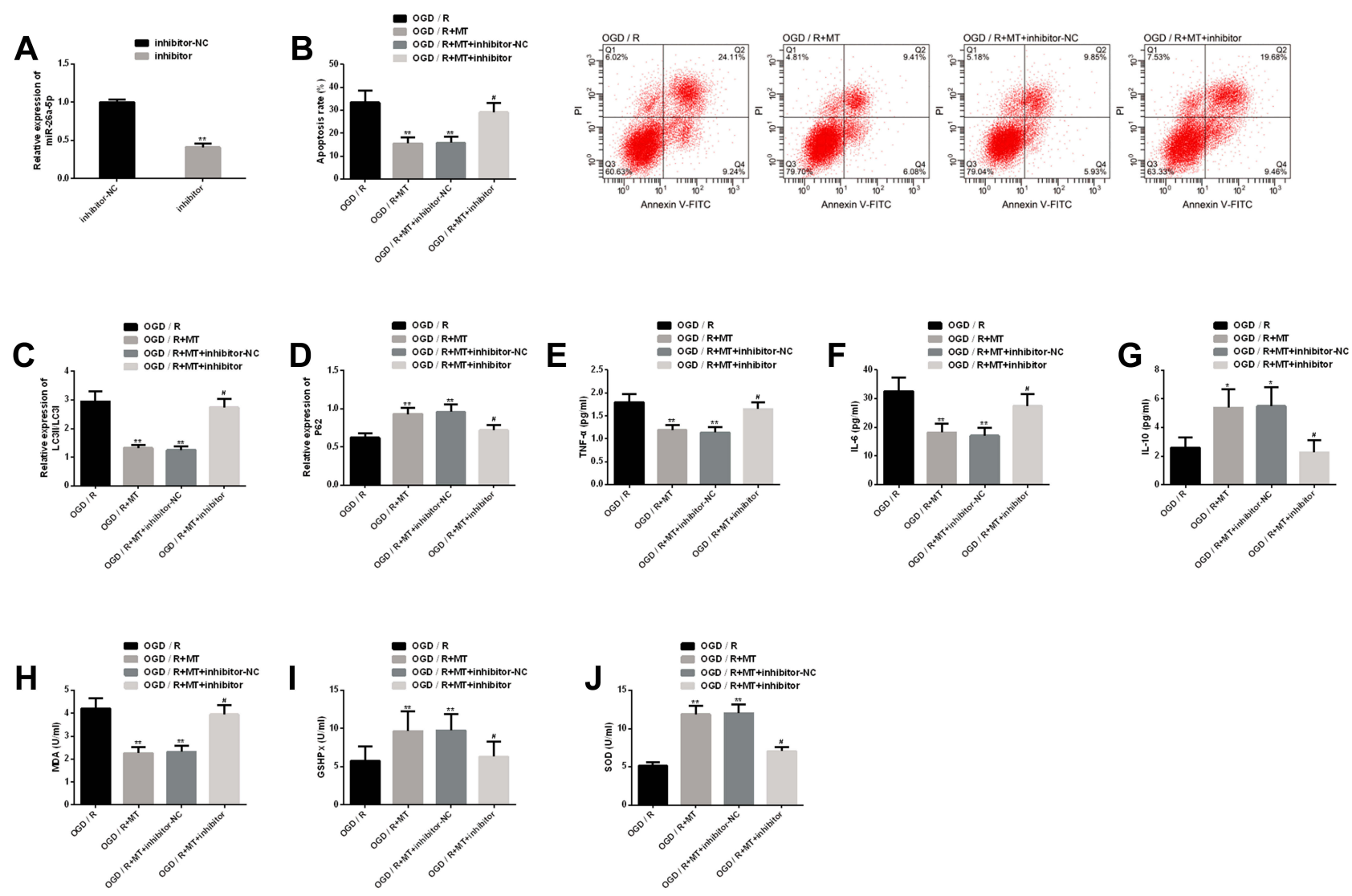

Figure 5 Interference of downregulation of miR-26a-5p on MT in vitro protection. (A) Transfection efficiency of miR-26a-5p; (B) Downregulating miR-26a-5p eliminates MT's inhibition on neuronal apoptosis rate and its flow cytometry is here; (C-D) Downregulating miR-26a-5p eliminates the improvement of MT on autophagy factor protein level; (E-G) Downregulating miR-26a-5p eliminates the improvement of MT on inflammatory indexes; (H-J) Downregulating miR-26a-5p eliminates the improvement of MT on oxidative stress.

Notes: Compared with OGD/R or inhibitor-NC, **represents $P<0.01$; compared with OGD/R, *represents $P<0.05$; compared with $O$ GD/R+MT, ${ }^{*}$ represents $P<0.05$. Abbreviations: MT, melatonin; miR, microRNA; OGD/R, oxygen-glucose deprivation/reperfusion; NC, negative control; PI, propidium iodide; FITC, fluorescein isothiocyanate; LC3, light chain 3; P62, sequestosome I; TNF, tumor necrosis factor; IL, interleukin; MDA, malondialdehyde; GSHPx, glutathione peroxidase; SOD, superoxide dismutase.

system diseases, such as repairing vascular cognitive impairment by activating ERK1/2 signaling pathway, and improving CIRI process by inhibiting endoplasmic reticulum stress and nerve cell apoptosis. ${ }^{19,25,26}$ In this study, we found that MT played an anti-CIRI protective role in vivo and in vitro by inhibiting neuronal apoptosis, autophagy, inflammation and oxidative stress.

MTs inhibition of CIRI neuronal apoptosis in vivo and in vitro may be related to autophagy inhibition. The autophagy process involves the formation and maturation of autophagy, accompanied by the transformation from LC3I to LC3II. Therefore, LC3II/LC3I index can be used to indicate the above process. ${ }^{27}$ P62 can be degraded by autophagy, and the main mechanism is to combine with autophagy membrane on LC3 to combine with autophagy. ${ }^{28}$ However, in our study, we observed a higher level of LC3II/LC3I and a lower level of P62 in CIRI models both in vivo and in vitro. The above situation was significantly improved after MT intervention. Zhang et $\mathrm{al}^{29}$ pointed out that astragaloside IV inhibited autophagy by downregulating LC3II/LC3I and upregulating P62, similar to our results. Feng et $\mathrm{al}^{30}$ also clarified that MT could inhibit endoplasmic reticulum stress-dependent autophagy by mediating PERK and IRE1 pathways, thus playing a neuroprotective role, which might partially explain the anti-autophagy mechanism of MT in this study. Besides, CIRI could stimulate dangerous molecular signals in the body, stimulate extracellular and intracellular pattern recognition receptor signals, thus inducing excessive release of inflammatory mediators, leading to neuronal apoptosis. ${ }^{31}$ TNF- $\alpha$ and IL- 6 are typical representatives of proinflammatory factors, which will aggravate the inflammatory injury of CIRI. IL-10 is an anti-inflammatory medium that helps prevent inflammation. All three are related to 

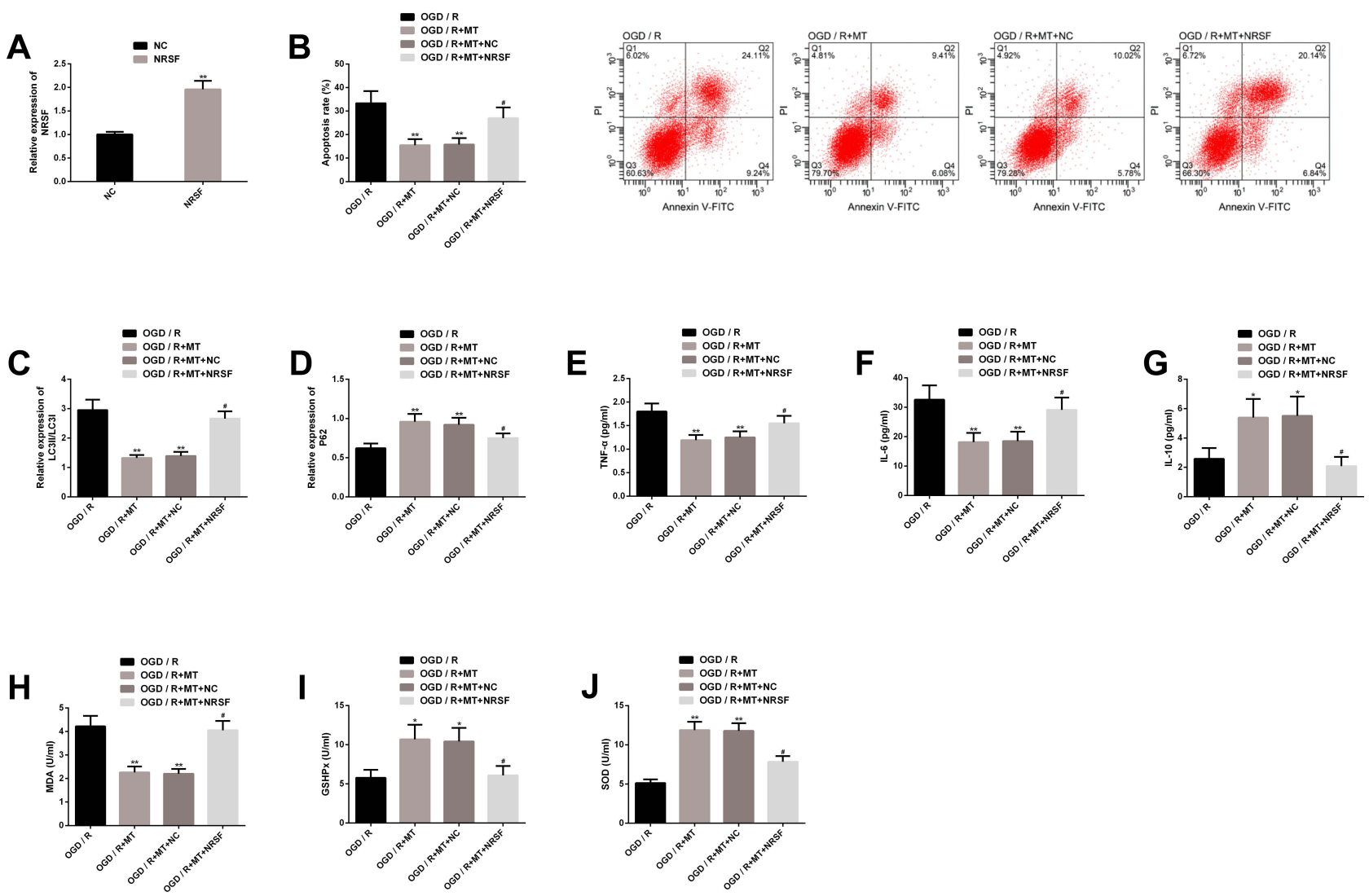

Figure 6 Upregulating the interference of NRSF on MT's in vitro protection. (A) Transfection efficiency of NRSF; (B) Upregulating NRSF eliminates inhibition of MT on neuronal apoptosis rate and its flow cytometry is here; (C-D) Upregulating NRSF eliminates the improvement of MT on autophagy factor protein level; (E-G) Upregulating NRSF eliminates the improvement of MT on inflammatory indexes; (H-J) Upregulating NRSF eliminates the improvement of MT on oxidative stress.

Notes: Compared with OGD/R or NC, **represents $P<0.01$; compared with OGD/R, *represents $P<0.05$; compared with $O G D / R+M T$, ${ }^{*}$ represents $P<0.05$.

Abbreviations: MT, melatonin; NRSF, neuron-restrictive silencing factor; OGD/R, oxygen-glucose deprivation/reperfusion; NC, negative control; PI, propidium iodide; FITC, fluorescein isothiocyanate; LC3, light chain 3; P62, sequestosome I; TNF, tumor necrosis factor; IL, interleukin; MDA, malondialdehyde; GSHPx, glutathione peroxidase; SOD, superoxide dismutase.

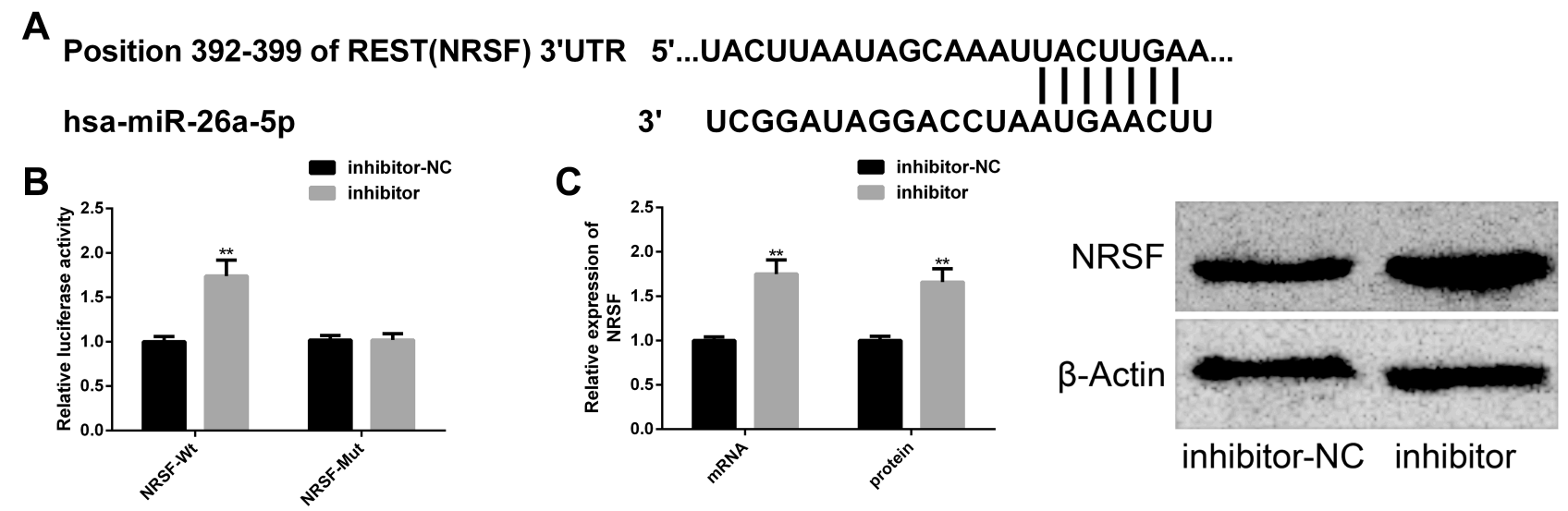

Figure 7 Targeted relationship between miR-26a-5p and NRSF. (A) Binding sites of miR-26a-5p and NRSF; (B) dual luciferase report; (C) miR-26a-5p can negatively regulate the mRNA and protein levels of NRSF and its protein map is here.

Notes: Compared with inhibitor-NC, ${ }^{*}$ represents $P<0.01$.

Abbreviations: miR, microRNA; NRSF, neuron-restrictive silencing factor; NC, negative control; Wt, wild type; Mut, mutant.

apoptosis or necrosis of neuronal cells during brain injury. ${ }^{32}$ In our research results, MT showed outstanding antiinflammatory effect in CIRI models both in vivo and in vitro, which effectively curbed the inflammatory imbalance in CIRI process. However, studies have shown that the anti-inflammatory mechanism of MT is related to the 

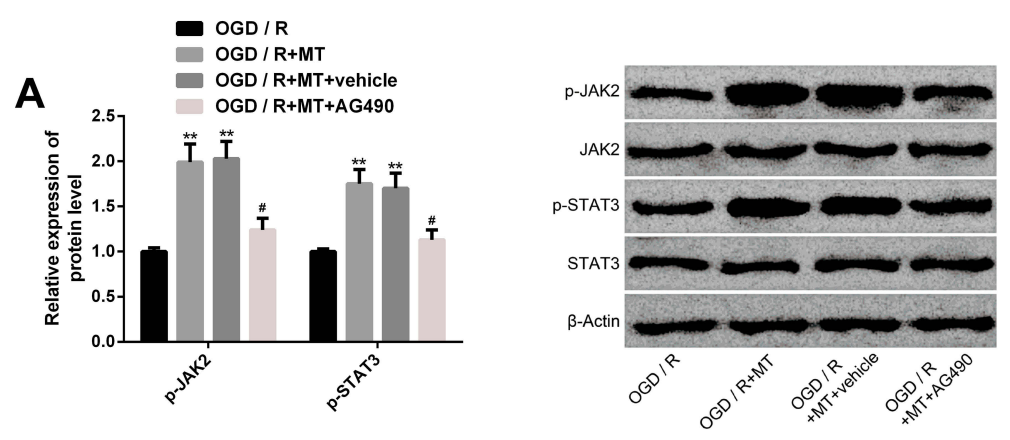

B
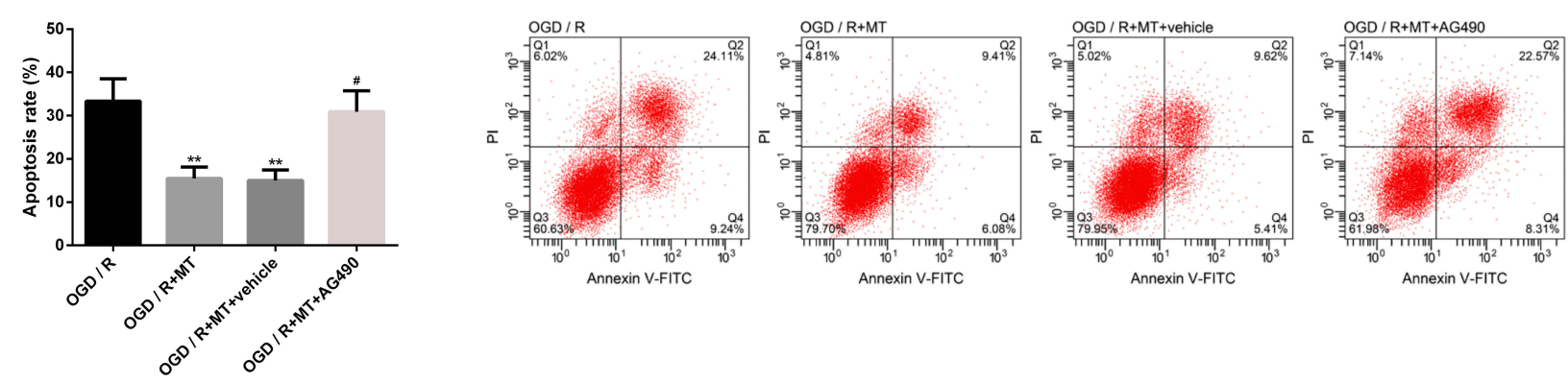

Figure 8 Effect of MT on JAK2-STAT3 pathway. (A) Effect of MT on the protein level of JAK2-STAT3 pathway marker and its protein map; (B) Effect of JAK2-STAT3 pathway inhibitor on MT antineuronal apoptosis and its cell flow diagram.

Notes: Compared with OGD/R, ${ }^{* *}$ represents $P<0.01$; compared with $\mathrm{OGD} / \mathrm{R}+\mathrm{MT}$, ${ }^{*}$ represents $P<0.05$.

Abbreviations: MT, melatonin; JAK2, Janus kinase-2; STAT3, signal transducer and activator of transcription-3; OGD/R, oxygen-glucose deprivation/reperfusion; PI, propidium iodide; FITC, fluorescein isothiocyanate; AG490, $\alpha$-cyano-(3,4-hydroxy) N-benzylstyramine.

upregulation of SIRT1 and the inhibition of activation of inflammatory signals such as mTOR and Notch by SIRT1. ${ }^{33}$ It is understood that inflammatory reaction and oxidative stress are interactive, and the latter may trigger cascade progression of CIRI and may cause malignant consequences such as brain death in serious cases. ${ }^{34}$ MDA was upregulated in CIRI oxidative stress injury, while the levels of antioxidant enzymes GSHPx and SOD were dramatically downregulated. ${ }^{35}$ The latter two are important components of antioxidant defense mechanism, which is conducive to the removal or reduction of peroxides. ${ }^{36}$ In this study, MT also showed encouraging antioxidant activity, whether in vivo or in vitro. Zeng et $\mathrm{al}^{5}$ confirmed that metformin could reduce oxidative stress of CIRI in vivo model by downregulating MDA level and upregulating GSHPx and SOD level, which was consistent with our research results. Chumboatong et $\mathrm{al}^{37}$ reported that MT receptor agonist (agomelatine) could inhibit oxidative stress by upregulating HO-1 and mediating Nrf2 signal pathway, thus playing an antioxidant role, which might be helpful to explain the antioxidant properties played by MT in this study.

We have proved the anti-autophagy, anti-inflammatory and anti-oxidation properties of MT in CIRI model, but the mechanism of miRNA-mRNA axis regulation is still unknown, so we have made further research. We found that miR-26a-5p and NRSF were both downregulated in CIRI in vivo and in vitro models, and MT could significantly reverse this abnormality. This leads us to suspect that the miR-26a-5p-NRSF axis may mediate the protective process of MT, and the results also confirm that downregulating miR-26a-5p or upregulating NRSF can eliminate the protective effect of MT as we think. Wei et $\mathrm{al}^{14}$ pointed out that miR-26a-5p was regulated by upstream molecule AK038897 and targeted downstream factor dAPK1, thus playing a role in CIRI. Other studies have confirmed that it is also involved in the pathological process of neuropathic pain and also presents a low level state in spinal cord tissue. It can inhibit the process of neuroinflammation and neuropathic pain by targeting MAPK6. ${ }^{38}$ NRSF is a vital factor that regulates neuron specificity. It exists widely in the brain and is relevant to the physiological processes of the nervous system. Its abnormal disorders are related to various brain diseases, such as brain tumors, mental disorders and CIRI. ${ }^{39}$ Luo et $\mathrm{al}^{40}$ explained that CIRI could strengthen nuclear NRSF-HDAC4 aggregation, suggesting that NRSF might accompany CIRI's pathological development. Zhang et al ${ }^{41}$ also reported that NRSF had a dual mechanism of promoting CIRI: One was to inhibit CART with neuroprotective effect by combining two components, and the other was to 
induce cell death by inhibiting CREB signal conduction activated by CART, which enlightened us that anti-NRSF preparations might be helpful for CIRI treatment. Further analysis found that miR-26a-5p had a definitely targeted relationship with NRSF, which was negatively regulated by the former, and whether it was mRNA level or protein level. What is more, we also confirmed that JAK2-STAT3 pathway also participated in the anti-CIRI effect of MT. Intervention with pathway inhibitor AG490 could significantly eliminate the antineuronal apoptosis of MT. Xu et $\mathrm{al}^{42}$ reported that $\mathrm{AG} 490$, as an inhibitor of JAK2STAT3 pathway, reversed the previous effect on the proliferation and apoptosis of retinoblastoma cells by inhibiting this pathway. The JAK2-STAT3 pathway is a signal transcription pathway that regulates gene expression and cell viability. It has been reported that it mediates the neuroprotective mechanism of hydroxyl-safflor yellow $A$ in focal cerebral ischemia and the protective effect of resveratrol on CIRI injury in ischemic stroke rats. ${ }^{43,44}$ Tang et $\mathrm{al}^{17}$ also reported that the JAK2-STAT3 pathway participated in the neuroprotective mechanism of CIRI, and the neuroprotective mechanism would collapse under the action of AG490.

Although this study has confirmed the anti-CIRI protective effect of MT and underlying mechanism, there is still room for improvement. First of all, we can supplement the upstream information molecules of miR-26a-5p, which is beneficial to successfully present the anti-CIRI protection mechanism of MT. Second, we can supplement antiendoplasmic reticulum stress of MT and other potential pathological defense effects, which helps us to further understand the pharmacological effects of MT. Furthermore, we can also increase the potential effect of JAK2-STAT3 pathway on MT in other CIRI pathological processes. Based on the above points, we will conduct supplementary research in the future.

\section{Conclusion}

In conclusion, we propose that MT plays a protective role by regulating miR-26a-5p-NRSF axis and JAK2-STAT3 pathway, thus improving CIRI autophagy, inflammation and oxidative stress, which may provide new insights for treating CIRI-related ischemic stroke and other central nervous system diseases.

\section{Funding}

There is no funding to report.

\section{Disclosure}

The authors report no conflicts of interest in this work.

\section{References}

1. Hou Y, Wang J, Feng J. The neuroprotective effects of curcumin are associated with the regulation of the reciprocal function between autophagy and HIF-1alpha in cerebral ischemia-reperfusion injury. Drug Des Devel Ther. 2019;13:1135-1144. doi:10.2147/DDDT.S194182

2. Rao JY, Wang Q, Wang YC, et al. Beta-caryophyllene alleviates cerebral ischemia/reperfusion injury in mice by activating autophagy. Zhongguo Zhong Yao Za Zhi. 2020;45(4):932-936. doi:10.19540/j.cnki.cjemm.20191112.402

3. Al-Senani F, Al-Johani M, Salawati M, et al. An epidemiological model for first stroke in Saudi Arabia. J Stroke Cerebrovasc Dis. 2020;29(1):104465. doi:10.1016/j.jstrokecerebrovasdis.2019.104465

4. Owjfard M, Bigdeli MR, Safari A, Namavar MR. Effects of nicorandil on neurobehavioral function, BBB integrity, edema and stereological parameters of the brain in the sub-acute phase of stroke in a rat model. J Biosci. 2020;45(1). doi:10.1007/s12038-020-0021-1

5. Zeng J, Zhu L, Liu J, et al. Metformin protects against oxidative stress injury induced by ischemia/reperfusion via regulation of the lncRNA-H19/miR-148a-3p/Rock2 axis. Oxid Med Cell Longev. 2019;2019:8768327. doi:10.1155/2019/8768327

6. Wongprayoon P, Govitrapong P. Melatonin receptor as a drug target for neuroprotection. Curr Mol Pharmacol. 2020. doi:10.2174/ 1874467213666200421160835

7. Tan HY, Ng KY, Koh RY, Chye SM. Pharmacological effects of melatonin as neuroprotectant in rodent model: a review on the current biological evidence. Cell Mol Neurobiol. 2020;40(1):25-51. doi:10.1007/s10571-019-00724-1

8. Liu L, Chen H, Jin J, et al. Melatonin ameliorates cerebral ischemia/ reperfusion injury through SIRT3 activation. Life Sci. 2019;239:117036. doi:10.1016/j.lfs.2019.117036

9. Wu XL, Lu SS, Liu MR, et al. Melatonin receptor agonist ramelteon attenuates mouse acute and chronic ischemic brain injury. Acta Pharmacol Sin. 2020. doi:10.1038/s41401-020-0361-2

10. Azedi F, Mehrpour M, Talebi S, et al. Melatonin regulates neuroinflammation ischemic stroke damage through interactions with microglia in reperfusion phase. Brain Res. 2019;1723:146401. doi:10.1016/ j.brainres.2019.146401

11. Xing J, Xu H, Liu C, et al. Melatonin ameliorates endoplasmic reticulum stress in N2a neuroblastoma cell hypoxia-reoxygenation injury by activating the AMPK-Pak2 pathway. Cell Stress Chaperones. 2019;24(3):621-633. doi:10.1007/s12192-019-00994-0

12. Di Y, Lei Y, Yu F, Changfeng F, Song W, Xuming M. MicroRNAs expression and function in cerebral ischemia reperfusion injury. $J \mathrm{Mol}$ Neurosci. 2014;53(2):242-250. doi:10.1007/s12031-014-0293-8

13. Potenza N, Mosca N, Mondola P, Damiano S, Russo A, De Felice B. Human miR-26a-5p regulates the glutamate transporter SLC1A1 (EAAT3) expression. Relevance in multiple sclerosis. Biochim Biophys Acta Mol Basis Dis. 2018;1864(1):317-323. doi:10.1016/j. bbadis.2017.09.024

14. Wei R, Zhang L, Hu W, Wu J, Zhang W. Long non-coding RNA AK038897 aggravates cerebral ischemia/reperfusion injury via acting as a ceRNA for miR-26a-5p to target DAPK1. Exp Neurol. 2019;314:100-110. doi:10.1016/j.expneurol.2019.01.009

15. Morris-Blanco KC, Kim T, Bertogliat MJ, Mehta SL, Chokkalla AK, Vemuganti R. Inhibition of the epigenetic regulator REST ameliorates ischemic brain injury. Mol Neurobiol. 2019;56(4):2542-2550. doi:10.1007/s12035-018-1254-y

16. Song Q, Liu B, Li X, et al. MiR-26a-5p potentiates metastasis of human lung cancer cells by regulating ITGbeta8- JAK2/STAT3 axis. Biochem Biophys Res Commun. 2018;501(2):494-500. doi:10.1016/j. bbrc.2018.05.020 
17. Tang Y, Tong X, Li Y, et al. JAK2/STAT3 pathway is involved in the protective effects of epidermal growth factor receptor activation against cerebral ischemia/reperfusion injury in rats. Neurosci Lett. 2018;662:219-226. doi:10.1016/j.neulet.2017.10.037

18. Han M, Hu L, Chen Y. Rutaecarpine may improve neuronal injury, inhibits apoptosis, inflammation and oxidative stress by regulating the expression of ERK1/2 and Nrf2/HO-1 pathway in rats with cerebral ischemia-reperfusion injury. Drug Des Devel Ther. 2019;13:2923-2931. doi:10.2147/DDDT.S216156

19. Lin YW, Chen TY, Hung CY, et al. Melatonin protects brain against ischemia/reperfusion injury by attenuating endoplasmic reticulum stress. Int J Mol Med. 2018;42(1):182-192. doi:10.3892/ijmm.2018.3607

20. Wang P, Jia L, Chen B, et al. Hydrogen inhalation is superior to mild hypothermia in improving cardiac function and neurological outcome in an asphyxial cardiac arrest model of rats. Shock. 2016;46 (3):312-318. doi:10.1097/SHK.0000000000000585

21. Ruscher K, Freyer D, Karsch M, et al. Erythropoietin is a paracrine mediator of ischemic tolerance in the brain: evidence from an in vitro model. J Neurosci. 2002;22(23):10291-10301. doi:10.1523/JNEUR OSCI.22-23-10291.2002

22. Jena I, Nayak SR, Behera S, et al. Evaluation of ischemia-modified albumin, oxidative stress, and antioxidant status in acute ischemic stroke patients. J Nat Sci Biol Med. 2017;8(1):110-113. doi:10.4103/ 0976-9668.198346

23. Wu H, Tang C, Tai LW, et al. Flurbiprofen axetil attenuates cerebral ischemia/reperfusion injury by reducing inflammation in a rat model of transient global cerebral ischemia/reperfusion. Biosci Rep. 2018;38 (4). doi:10.1042/BSR20171562

24. Kuo MH, Lee HF, Tu YF, Lin LH, Cheng YY, Lee HT. Astaxanthin ameliorates ischemic-hypoxic-induced neurotrophin receptor p75 upregulation in the endothelial cells of neonatal mouse brains. Int J Mol Sci. 2019;20(24). doi:10.3390/ijms20246168

25. Zhang R, Wang X, Ni L, et al. COVID-19: melatonin as a potential adjuvant treatment. Life Sci. 2020;250:117583. doi:10.1016/j. lfs. 2020.117583

26. Chen BH, Park JH, Lee YL, et al. Melatonin improves vascular cognitive impairment induced by ischemic stroke by remyelination via activation of ERK1/2 signaling and restoration of glutamatergic synapses in the gerbil hippocampus. Biomed Pharmacother. 2018;108:687-697. doi:10.1016/j.biopha.2018.09.077

27. Oh SH, Choi YB, Kim JH, Weihl CC, Ju JS. Quantification of autophagy flux using LC3 ELISA. Anal Biochem. 2017;530:57-67. doi:10.1016/j.ab.2017.05.003

28. Wang J, Garbutt C, Ma H, et al. Expression and role of autophagy-associated p62 (SQSTM1) in multidrug resistant ovarian cancer. Gynecol Oncol. 2018;150(1):143-150. doi:10.1016/j. ygyno.2018.04.557

29. Zhang Y, Zhang Y, Jin XF, et al. The role of astragaloside IV against cerebral ischemia/reperfusion injury: suppression of apoptosis via promotion of P62-LC3-autophagy. Molecules. 2019;24(9):1838.

30. Feng D, Wang B, Wang L, et al. Pre-ischemia melatonin treatment alleviated acute neuronal injury after ischemic stroke by inhibiting endoplasmic reticulum stress-dependent autophagy via PERK and IRE1 signalings. J Pineal Res. 2017;62(3):e12395. doi:10.1111/jpi.12395
31. Ma $\mathrm{C}$, Wang $\mathrm{X}, \mathrm{Xu}$ T, et al. Qingkailing injection ameliorates cerebral ischemia-reperfusion injury and modulates the AMPK/ NLRP3 inflammasome signalling pathway. BMC Complement Altern Med. 2019;19(1):320. doi:10.1186/s12906-019-2703-5

32. Lv Z, Liu C, Zhai M, et al. LPS pretreatment attenuates cerebral ischaemia/reperfusion injury by inhibiting inflammation and apoptosis. Cell Physiol Biochem. 2018;45(6):2246-2256. doi:10. $1159 / 000488170$

33. Hardeland R. Melatonin and inflammation-story of a double-edged blade. J Pineal Res. 2018;65(4):e12525. doi:10.1111/jpi.12525

34. Wu L, Xiong X, Wu X, et al. Targeting oxidative stress and inflammation to prevent ischemia-reperfusion injury. Front Mol Neurosci. 2020;13:28. doi:10.3389/fnmol.2020.00028

35. Maslov LN, Naryzhnaia NV, Podoksenov IK, et al. Reactive oxygen species are triggers and mediators of an increase in cardiac tolerance to impact of ischemia-reperfusion. Ross Fiziol Zh Im I M Sechenova. 2015;101(1):3-24.

36. Wang FJ, Wang SX, Chai LJ, Zhang Y, Guo H, Hu LM. Xueshuantong injection (lyophilized) combined with salvianolate lyophilized injection protects against focal cerebral ischemia/reperfusion injury in rats through attenuation of oxidative stress. Acta Pharmacol Sin. 2018;39(6):998-1011. doi:10.1038/aps.2017.128

37. Chumboatong W, Thummayot S, Govitrapong P, Tocharus C, Jittiwat J, Tocharus J. Neuroprotection of agomelatine against cerebral ischemia/reperfusion injury through an antiapoptotic pathway in rat. Neurochem Int. 2017;102:114-122. doi:10.1016/j.neuint. 2016.12.011

38. Zhang Y, Su Z, Liu HL, et al. Effects of miR-26a-5p on neuropathic pain development by targeting MAPK6 in in CCI rat models. Biomed Pharmacother. 2018;107:644-649. doi:10.1016/j.biopha.2018.08.005

39. Zhao Y, Zhu M, Yu Y, et al. Brain REST/NRSF is not only a silent repressor but also an active protector. Mol Neurobiol. 2017;54 (1):541-550. doi:10.1007/s12035-015-9658-4

40. Luo P, Fu X, Chang M, Zhang L, Guo L. Cerebral ischemia-reperfusion causes a down regulation of $\mathrm{HCN} 1$ expression via enhancing the nuclear NRSF-HDAC4 gathering that contributes to neuron damage. Brain Res Bull. 2020;156:50-57. doi:10.1016/j. brainresbull.2020.01.001

41. Zhang J, Wang S, Yuan L, et al. Neuron-restrictive silencer factor (NRSF) represses cocaine- and amphetamine-regulated transcript (CART) transcription and antagonizes cAMP-response element-binding protein signaling through a dual NRSE mechanism. $J$ Biol Chem. 2012;287 (51):42574-42587. doi:10.1074/jbc.M112.376590

42. Xu B, Chen X, Tan J, Xu X. Effect of AG490 on JAK2/STAT3 signaling pathway in human retinoblastoma HXO-RB44 cell lines. Zhong Nan Da Xue Xue Bao Yi Xue Ban. 2018;43(10):1061-1067. doi:10.11817/j.issn.1672-7347.2018.10.004

43. Hou Y, Wang K, Wan W, Cheng Y, Pu X, Ye X. Resveratrol provides neuroprotection by regulating the JAK2/STAT3/PI3K/AKT/mTOR pathway after stroke in rats. Genes Dis. 2018;5(3):245-255. doi:10.1016/j.gendis.2018.06.001

44. Yu L, Liu Z, He W, et al. Hydroxysafflor yellow a confers neuroprotection from focal cerebral ischemia by modulating the crosstalk between JAK2/STAT3 and SOCS3 signaling pathways. Cell Mol Neurobiol. 2020. doi:10.1007/s10571-020-00812-7

\section{Dovepress}

\section{Publish your work in this journal}

Drug Design, Development and Therapy is an international, peerreviewed open-access journal that spans the spectrum of drug design and development through to clinical applications. Clinical outcomes, patient safety, and programs for the development and effective, safe, and sustained use of medicines are a feature of the journal, which has also been accepted for indexing on PubMed Central. The manuscript management system is completely online and includes a very quick and fair peer-review system, which is all easy to use. Visit http://www. dovepress.com/testimonials.php to read real quotes from published authors. 